\title{
A model for evaluating the effect of fatigue crack repair by the infiltration method
}

\author{
C. S. SHIN and C. Q. CAI \\ Department of Mechanical Engineering, National Taiwan University, No. 1, Sec. 4, Roosevelt Road, Taipei, 10617, Taiwan, Republic of China \\ Received in final form 10 fuly 2000
}

\begin{abstract}
Infiltration of foreign materials into a fatigue crack has previously been shown to be able to retard the crack and extend fatigue life. Most of the related studies were empirical and phenomenological in nature. To aid engineering decisions, it would be advantageous if the possible outcome of a repair can be evaluated beforehand. To this end, a crack closure model taking into account the additional closure effect of the infiltrant has been developed and verified against experimental results. With this model, the sensitivity of the repair effect to various parameters such as mechanical properties of the infiltrant, depth of penetration and infiltration load level can be assessed.
\end{abstract}

Keywords closure model; crack arrest; crack infiltration; fatigue crack closure; fatigue damage repair.

\section{INTRODUCTION}

Fatigue fracture accounts for a great number of mechanical failures. When a fatigue crack is discovered in a critical component during routine maintenance, replacement of the component may not be feasible as there may not be stocks or the available downtime is too limited. Some expeditious provisory fixing may then be needed to allow the component to operate safely until a more elaborate repair or replacement can be arranged. Infiltration of foreign materials into a fatigue crack has been shown to be able to bring about additional crack closure and subsequent crack growth retardation. ${ }^{1-3}$ This effect has been used to extend fatigue life. ${ }^{4-6}$ Different infiltration materials have been tested and effective infiltration methods have been developed..$^{7-11}$ The development of this technique has recently been reviewed. ${ }^{12}$ The amount of retardation achieved by infiltration is strongly influenced by the properties of the infiltrant as well as how effectively the infiltrant penetrates into a crack. The depth of infiltrant penetration depends on the fatigue loading condition and the load level at which infiltration is being carried out.

Correspondence: C. S. Shin, Department of Mechanical Engineering, National Taiwan University, No. 1, Sec. 4, Roosevelt Road, Taipei 10617, Taiwan, Republic of China.

E-mail: csshin@ccms.ntu.edu.tw
Most of the related studies were empirical and phenomenological in nature. For practical application, it would be advantageous if the possible outcome of a repair can be evaluated beforehand.

A simplified model assuming a uniform infiltrant contact stress ${ }^{13}$ met with limited success. Researchers Ur-Rehman and Thomason ${ }^{5,14}$ argued that due to small deformation and large plastic constraint, a low-strength elastic wedge will behave as nearly rigid. However, on taking into account the contribution of this wedge to the crack closure, they found that the cracks under investigation should have arrested, which was contrary to what they actually observed. At crack lengths sufficiently far away from the wedge tip, crack growth was predicted to occur, but the predicted rates were lower than observed. Ur-Rehman and Thomason attributed the differences between the observed and predicted growth behaviour to: (i) a finite crack length effect; (ii) an irregularity in wedge geometry; and (iii) friction between the wedge and crack interfaces. Careful experimental examination showed that a deduced rigid wedge that suits the retardation behaviour differs vastly in size and location to the measured wedge. ${ }^{8}$

In the current work, a crack closure model embodying an elastic-perfectly-plastic infiltrant has been established. With this model, it is possible to evaluate the amount of crack closure brought about by different infiltration conditions. It is hoped that the 
simulated results can shed more light on the effect of infiltrating foreign materials with different mechanical properties, under different infiltration load levels, different fatigue loading ratios and to different depths of infiltrant penetration.

\section{CRACK CLOSURE MODEL}

An infiltrated wedge will bring about premature contact of the crack flanks and so react to part of the applied loading. As a result the effective crack driving force will be reduced and crack growth will be retarded. The current model attempts to predict this crack driving force reduction from the geometrical interference that causes premature contact and reaction stresses in the wedge. The infiltrants used (mainly polymers) in a number of experiments have much lower Young's moduli and yield strengths than the specimen materials (mainly engineering alloys). Hence in this model, for simplicity, we assume during the calculations that all deformation occurs in the wedge. Moreover, the wedge materials are assumed to be elastic-perfectly-plastic. Figure 1 shows schematically the development of crack closure for the upper half of an infiltrated crack. In Fig. 1(a), the crack of length $a$ is propped open at the infiltration stress level (ISL) and a wedge that spans the region from $b_{1}$ to $b_{2}$ is snugly placed into the crack. Also shown on the crack
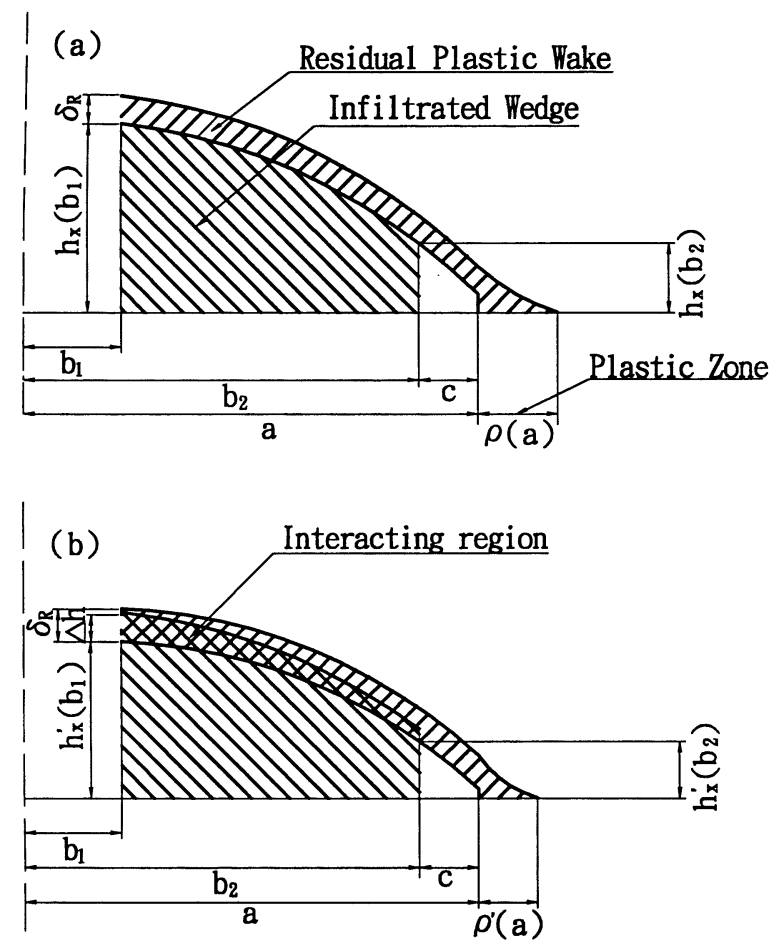

surface is a layer of residual plastic wake left over by previous plastic deformation at the crack tip. Figure 1(b) shows the same crack at the minimum applied loading $K_{\text {min }}$. The thickness $b_{x}$ of the infiltrated wedge is now compressed and deformed elastic-plastically to $b_{x}^{\prime}$ along the crack length. Reactive stresses varying according to the amount of deformation which will arise in the wedge and act on the crack surface. This wedge-opening action will lead to an opening stress intensity $K_{\text {wedge }}$. To remove surface contact and allow the crack to become fully open again, a stress intensity increment equal to $K_{\text {wedge }}$ has to be applied. Hence the fully open stress intensity level, denoted as $K_{\text {op, }}$ may be calculated by:

$K_{\text {op }}=K_{\text {min }}+K_{\text {wedge }}$

On loading up again, elastic recovery of the wedge will occur. In Fig. 1(c) the crack has increased in length to $(a+\Delta a)$, while the wedge has undergone plastic deformation and subsequent elastic recovery to thickness $b_{x-\text { rec }}$. On unloading the specimen again, only part of the wedge will now come into contact with the crack surface [Fig. 1(d)]. The corresponding opening stress intensity level can again be calculated by Eq. (1).

The compressive deformation of the wedge at various positions of the crack may be deduced from a comparison of the crack opening displacement (COD) at the opening stress intensity level and the corresponding unloaded
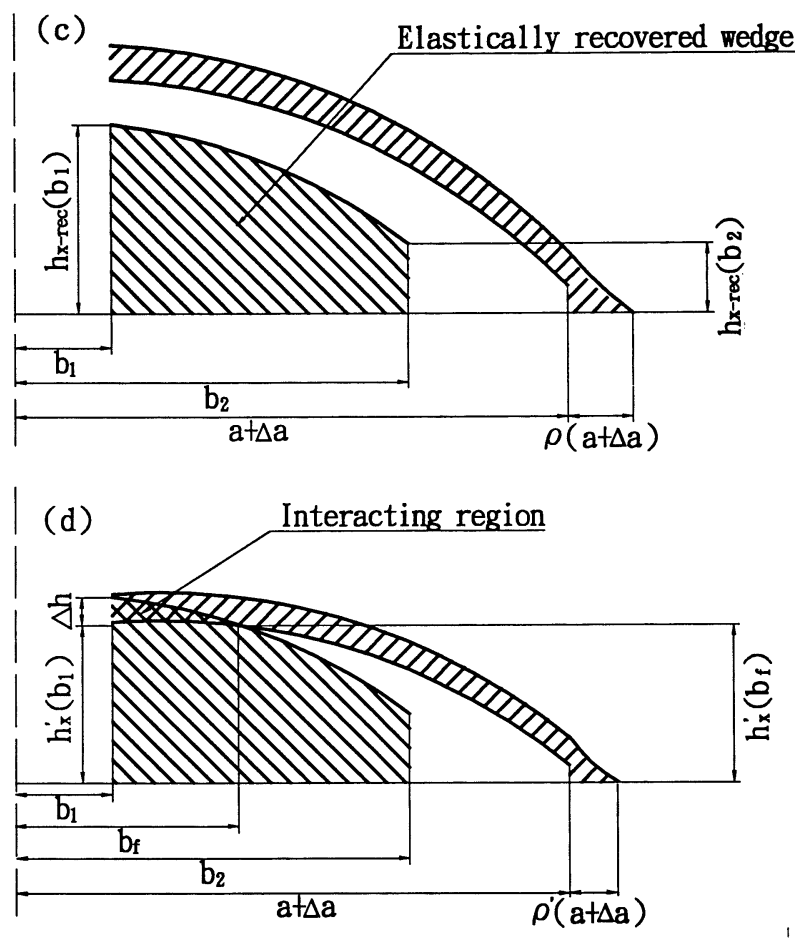

Fig. 1 Schematic of the development of crack closure induced by an infiltrated wedge. (a) just after infiltration at the prop-opening load; (b) contact interference developed on unloading to minimum load; (c) crack growth occurred after infiltration; (d) contact interference developed on unloading after some crack growth. 
wedge thickness. By cutting up the wedge into a number of strips, the distribution of the reactive wedge stress along the crack can be deduced from the corresponding deformation. Using a suitable weight function, $K_{\text {wedge }}$ can be calculated from this stress distribution. In Eq. (1), both $K_{\mathrm{op}}$ and $K_{\text {wedge }}$ are unknown. An iterative procedure can be set up to first-guess the value of $K_{\mathrm{op}}$. By using the above procedures, $K_{\text {wedge }}$ can be calculated and an updated $K_{\text {op }}$ guess be obtained. The process is repeated until $K_{\text {op }}$ and $K_{\text {wedge }}$ converge to constant values.

Since most of the available experimental results were derived from compact tension specimens, in this work the corresponding weight functions derived by Mall and Newman ${ }^{15}$ have been used for calculating the stress intensity and COD under pin loading and under a crack surface stress distribution. The weight functions are reproduced in the Appendix. For other specimen geometries, the relevant weight functions should be used. In calculating the net CODs, the thickness of the residual plastic wake has been deducted from the linear elastic fracture mechanics COD values. The thickness of the residual plastic wake is approximated by the result given by Budiansky \& Hutchinson ${ }^{16}$ :

$\frac{\delta_{\text {tip }}-\delta_{\mathrm{R}}}{\delta_{\text {tip }}}=0.5\left(\frac{K_{\max }-K_{\mathrm{op}}}{K_{\max }}\right)^{2}$

where $K_{\max }$ is the maximum stress intensity of the loading cycle, $\delta_{\text {tip }}$ is the crack tip opening displacement at $K_{\max }$, and $\delta_{\mathrm{R}}$ is the thickness of the residual plastic wake.

\section{RESULTS AND DISCUSSION}

From a consideration of the above model, it is clear that the amount of retardation brought about by infiltration of foreign material is affected by a number of factors. These include: the range of crack length coverage, the fill-up percentage of the crack opening displacement at the infiltration load, the infiltration stress level and the deformation properties such as Young's modulus and yield strength of the infiltrant. Moreover, the fatigue loading ratio $R$ ( $\mathrm{min}$. load/max. load) acting on the specimen may also affect the outcome. These effects will now be examined in the following using a compact tension specimen of width $50 \mathrm{~mm}$ containing a $20 \mathrm{~mm}$ crack. The specimen material is taken as AISI 304 stainless steel which has a yield strength and tensile strength of 375 and $665 \mathrm{MPa}$, respectively. The crack growth rate $(\mathrm{d} a / \mathrm{d} N)$ and the effective stress intensity range $\left(\Delta K_{\mathrm{eff}},=K_{\max }-K_{\mathrm{op}}\right)$ is related by:

$\frac{\mathrm{d} a}{\mathrm{~d} N}=4.6093 \times 10^{-8}\left(\Delta K_{\mathrm{eff}}\right)^{2.479}$
Unless otherwise stated, the test conditions for calculation are a constant applied stress intensity range of $25 \mathrm{MPa} \sqrt{\mathrm{m}}$ at an $R$ ratio of 0.1 . Infiltration was made at the maximum cyclic load with an infiltrant that has a yield strength of $70 \mathrm{MPa}$ and a Young's modulus of $1.63 \mathrm{GPa}$. Lastly, a comparison between the model prediction and some experimental results will be made.

\section{Effect of crack length coverage}

During the early stage of development, the infiltration technique was not efficient and the infiltrant only penetrated to a few millimetres behind the crack tip. Even with a more efficient infiltration method, thorough penetration may not be possible if the infiltration stress level is too low, the viscosity or surface tension of the infiltrant too high or if the infiltrant contains solid particles. ${ }^{8}$

Figure 2(a) shows the predicted growth responses following infiltration at the maximum cyclic load for various crack length coverage. Infiltrant coverage starts from the tip of the starter notch $(15 \mathrm{~mm})$ and extends to various points behind the crack tip $(20 \mathrm{~mm})$. It can be seen that a coverage from 15 to $17 \mathrm{~mm}$ caused minimal retardation in growth rate. Significant retardation (greater than one order of magnitude) starts to occur only if the infiltrant coverage is within $1 \mathrm{~mm}$ behind the crack tip. The closer to the crack tip, the more serious is the initial retardation. Due to the occurrence of a singularity in computation, the effect of filling right up to the crack tip can only be approximated by filling up to $19.9999 \mathrm{~mm}$. In this case, a retardation of more than two orders of magnitude is obtained. At the current infiltration stress level, the retardation responses for coverage to $19.5 \mathrm{~mm}$ and to $20 \mathrm{~mm}$ do not show much difference. In all the cases considered, crack growth can still occur despite the various degrees of retardation. Moreover, the difference in the amount of retardation rapidly decreases as the crack continues to grow.

\section{Effect of infiltration stress level}

Most of the experiments on infiltration were carried out with the specimens held open at a certain static load. In this article, the infiltration stress level (ISL) is represented as a fraction of the maximum stress intensity $K_{\max }$. Figure 2(b) compares the effect of retardation at different ISL values. The infiltrant coverage is assumed to be from 15 to $19.5 \mathrm{~mm}$ (i.e. up to $0.5 \mathrm{~mm}$ behind the crack tip). At an ISL of 0.7 , the initial retardation is less than one order of magnitude. At 0.8 , initial retardation is nearly by one order. As the ISL increases from 0.7 to 1.0 , the amount of initial retardation showed marked increases. However, above 1.0, the increase in ISL does 


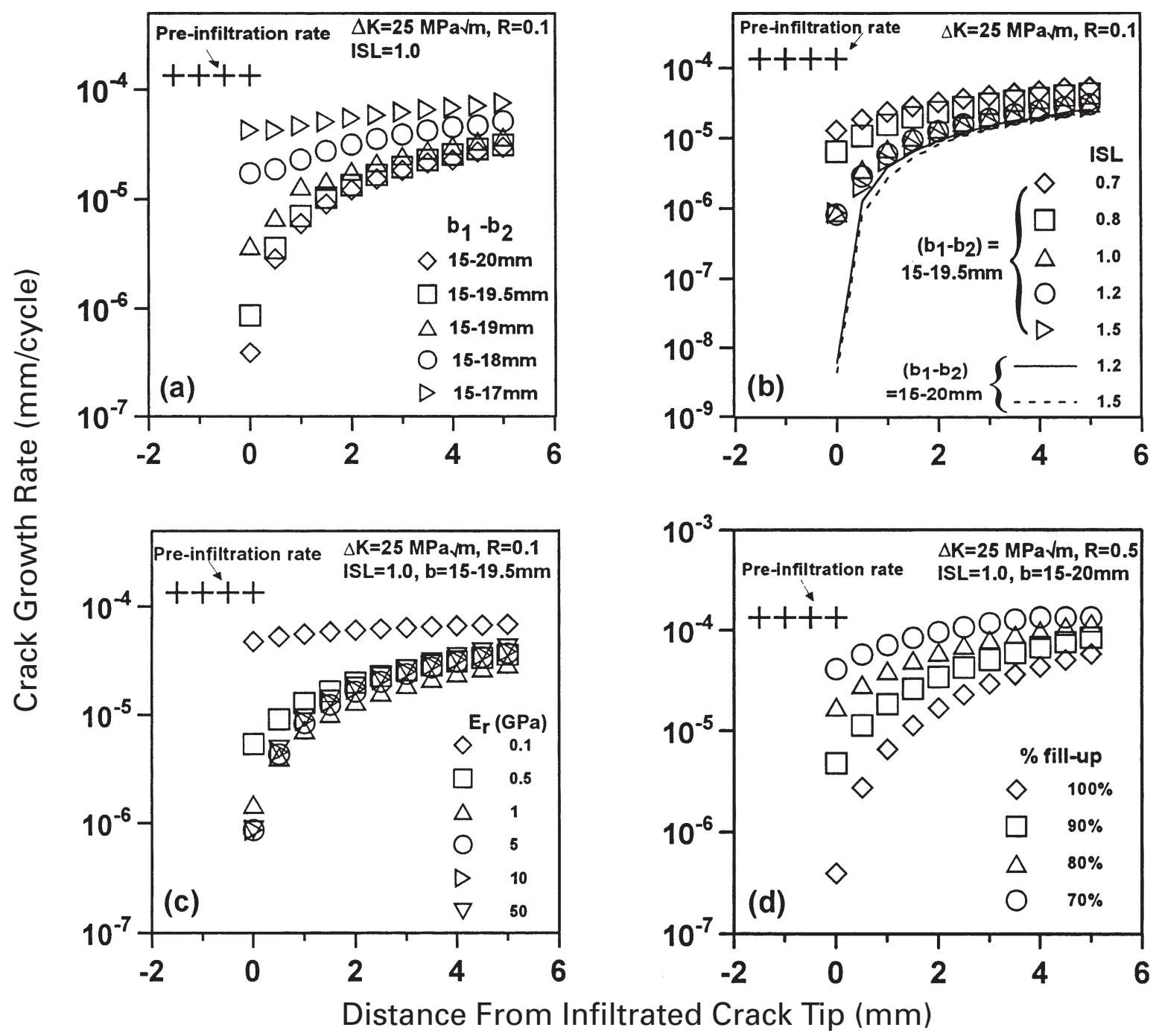

Fig. 2 Development of crack growth responses following infiltration (a) for various crack length coverage of the infiltrant; (b) at different infiltration stress levels (ISL); (c) with infiltrants of different stiffness; (d) for different infiltrant fill-up percentages.

not cause any significant additional increase in the retardation. As the crack continued to grow, the difference in the subsequent retardation responses for the different ISL values becomes smaller.

It should be pointed out that with ISL above 1.0, two effects may become significant. First, the 'overload' alone will retard the crack. This will be added to the retardation effect from the infiltrated wedge. Previous work on AISI 304 stainless steel showed that under similar applied loading, ISL values of 1.2 and 1.5 retarded the growth to 67 and $33 \%$ of the baseline rate. These are small even when compared with the retardation derived from infiltration at an ISL of 0.7 . Moreover, the residual plastic wake of the overload becomes fully developed and brings about retardation only when the crack grows into the overload plastic zone. Occurrence of an infiltrated wedge tends to prop-open the crack and prevents the full effect of the overload plastic wake being exerted. Hence in the current work the overload retardation effect has been neglected in the calculations. The second effect of an ISL above 1.0 is that the crack is now propped open to a wide slit. The infiltrant now stands a good chance of getting very close to the crack tip and the amount of infiltrant coverage is likely to increase. On the other hand, with an ISL of 0.7 or 0.8 , it is doubtful whether the infiltrant can get within $0.5 \mathrm{~mm}$ behind the crack tip. With these two effects in mind, it is expected that the actual difference in retardation responses should be even more prominent than that shown in Fig. 2(b).

For comparison, the retardation responses for ISL above 1.0 with infiltrant coverage up to the crack tip are also shown as lines in Fig. 2(b). In these cases, the initial 
retardation amounts to four orders of magnitude and crack arrest is likely to occur. This is in marked contrast with the case of ISL equal to 1.0, where coverage to $19.5 \mathrm{~mm}$ and $20 \mathrm{~mm}$ do not give significantly different responses [see Fig. 2(a)]. Interestingly, if the crack continues to grow, the subsequent retardation responses do not differ much from the lower ISL value and lower infiltrant coverage cases.

\section{Effect of Young's modulus of infiltrant}

Figure 2(c) compares the growth responses resulted from infiltrants with a Young's modulus $\left(E_{\mathrm{r}}\right)$ ranging from $0.1 \mathrm{GPa}$ to $50 \mathrm{GPa}$. With the ultra low modulus of $0.1 \mathrm{GPa}$, nearly no retardation is obtained. The amount of retardation increases significantly as the infiltrant modulus increases through $0.5 \mathrm{GPa}$ up to $1 \mathrm{GPa}$. On increasing the infiltrant modulus further to $5 \mathrm{GPa}$, the initial retardation increases while the subsequent retardation decreases, both by a small amount. Finally, above $5 \mathrm{GPa}$, an increase in infiltrant modulus caused no significant difference in the retardation behaviour.

\section{Effect of fill-up percentage}

It has been observed that when the crack opening displacement during infiltration is large, infiltrants with good flowability (such as an uncured epoxy resin and a molten solder) tended to seep out from the widely opened crack before they hardened. As a result, the infiltrated wedge thickness is only a fraction of the crack opening profile at the infiltration load. Figure 2(d) demonstrates that at a stress intensity range $\Delta K$ of $25 \mathrm{MPa} \sqrt{\mathrm{m}}$ and an $R$ ratio of 0.5 , a decrease in fill-up percentage from 100 to $80 \%$ reduced the amount of initial retardation by more than one order of magnitude. Significant reduction persisted even when the crack continued to grow. On the other hand, a decrease in fill-up percentage from 80 to $70 \%$ induced a much smaller reduction in the amount of crack growth retardation.

\section{Effect of infiltrant yield strength}

Ur-Rehman and Thomason ${ }^{5,14}$ argued that a huge plastic constraint would be imposed on the thin wedge infiltrated inside a crack. Moreover, the elastic deformation of the wedge is also very small. As a result they suggested that a material which yields at a value as low as $10 \mathrm{MPa}$ would appear as virtually rigid. Experimental work ${ }^{17}$ showed that infiltrants with different yield strengths produced significantly different amounts of retardation. Recent finite element results ${ }^{17}$ suggest that plastic constraint only raised the equivalent yield strength to about three times the uniaxial value. The current work also employed a constraint factor of 3 for the plastic deformation of the infiltrated wedge.

Figure 3(a) shows the predicted retardation responses for different infiltrant yield strengths using the current model at $R=0.1$ and an ISL of 1.0. Infiltrant coverage is assumed to be from 15 to $19.5 \mathrm{~mm}$. There is an obvious increase in the amount of retardation as the infiltrant yield strength increases. For yield strengths of 10 and $25 \mathrm{MPa}$, there is only a minimal retardation. As the yield strength increases to $50 \mathrm{MPa}$, the initial retardation amounts to one order of magnitude. The increase in the initial retardation is more marked than that in the subsequent growth as infiltrant yield strength increases. Figure 3(b) shows the effect of infiltrant yield strength when the ISL is raised to 1.5 . Infiltrant coverage for these cases has been changed to 15 to $20 \mathrm{~mm}$. The trends described above for ISL $=1.0$ are still valid and the difference in retardation responses brought about by the difference in yield strength is even more prominent.

\section{Effect of load ratio}

Figure 3(c) compares the retardation responses under different $R$ ratios at two different values of ISL. For an ISL of 1.0, the retardation responses for $R$ ranging from 0.1 to 0.5 showed no marked distinction. However, careful examination shows that the initial retardation at $R=0.3$ is slightly lower than that for $R=0.1$. The trend reverses as $R$ increases to 0.5 . At $R$ equals 0.7 , a markedly higher initial retardation occurred. As the crack continued to grow, the relative amount of retardation among different $R$ ratios changes. Subsequently the amount of retardation is less for a higher value of $R$. These trends in the retardation development at different $R$ ratios also hold true and in fact become more prominent for ISL values of 1.2 and 1.5. The development of retardation at different values of $R$ for ISL $=1.5$ has been included in Fig. 3(c) for comparison. At a constant ISL, a higher $R$ ratio will result in a larger crack opening. This will enable a thicker wedge to be infiltrated yet produce a smaller amount of contact interference. These two phenomena have opposite effects on the amount of induced crack closure, giving rise to the trends observed above.

\section{Effect of applied stress intensity range}

Figure 3(d) compares the retardation responses resulting from infiltration under applied values of $\Delta K$ of $3.5,9$ and $22 \mathrm{MPa} \sqrt{\mathrm{m}}$ at a load ratio of 0.1 . These correspond to pre-infiltration growth rates of $10^{-4}, 10^{-5}$ and $10^{-6} \mathrm{~mm} /$ cycle, respectively. After infiltration at an ISL $=1.0$, the initial growth rates all fell by more than two orders of magnitude. 


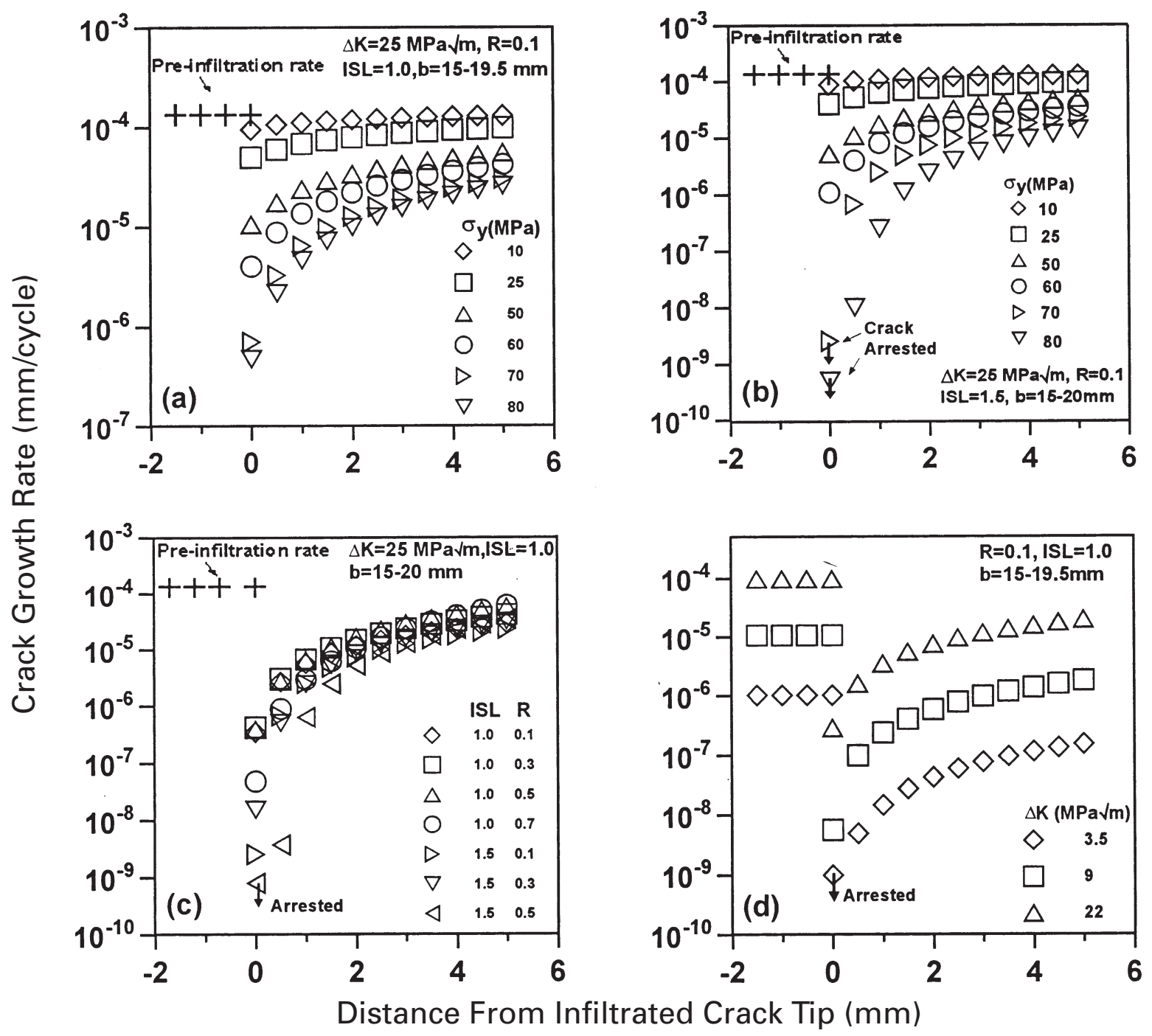

Fig. 3 Development of crack growth responses following infiltration (a) with infiltrants of different yield strengths at ISL $=1.0$; (b) with infiltrants of different yield strengths at ISL $=1.5$; (c) at different $R$ ratios; (d) under different applied values of $\Delta K$.

The maximum amount of initial retardation was obtained for the case with a pre-infiltration growth rate of $10^{-6} \mathrm{~mm} /$ cycle ( $>4$ orders). Since the retarded rate is below $10^{-10} \mathrm{~mm} /$ cycle, crack arrest is considered to have occurred. The initial retardation for the $10^{-5} \mathrm{~mm} /$ cycle case $(\sim 3.3$ orders $)$ comes second and finally it is the $10^{-4} \mathrm{~mm} /$ cycle case ( $\sim 2.5$ orders). Assuming crack growth can continue, the crack growth increments for regaining half of the growth rates on the $\log$ scale for the three cases are $0.74,1.0$ and $1.4 \mathrm{~mm}$, respectively.

Based on the above, a crack with a lower pre-infiltration growth rate has a better chance of getting arrested by infiltration. However, it should be noted that the above discussion assumed that the infiltrant has gone to the same vicinity (within $0.5 \mathrm{~mm}$ ) of the crack tip at all load levels. In practice, as the applied loading gets smaller, the crack opening also decreases accordingly and so infiltration may not be as perfect as that for a larger crack opening. The actual amount of retardation for lower load levels may be less than that presented in Fig. 3(d).

\section{Comparison with experimental data}

A number of results of crack-infiltration-induced crack growth retardation has been collected from the literature and checked against computed results using the above model. For experiments carried out in our own laboratory, we have tried to measure Young's modulus and yield strength on compression of the infiltrants as far as possible. $^{8,17}$ Other literature may not have reported 
these properties for the infiltrants concerned. Furthermore, information about the proximity of infiltrant to the crack tip is often lacking. Reasonable estimates for these values have to be employed, which may lead to uncertainty in the following computations.

Figure 4(a) is the retardation responses due to the infiltration of epoxy resin. ${ }^{8}$ The specimen material was 304 stainless steel. The $R$ ratio was 0.1 and the ISL was 0.95 . A proximity to crack tip of $0.55 \mathrm{~mm}$ was assumed based on observation of the fracture surface. The experiment was repeated thrice. It has been pointed out in Shin et $a l^{8}$ that experimental scatter occurred due to a varying degree of effectiveness in infiltration. This explanation is in line with the fact that the predicted response forms a lower bound and is approached by two of the tests (CT1 and CT3).

Figure 4(b) depicts the result ${ }^{17}$ for a similar experimental condition to Fig. 4(a), except that the $R$ ratio is now 0.3 . The measured retardation is about an order of magnitude larger than predicted when a $100 \%$ fill-up was assumed. In this work, a tunnel was drilled through the height of the specimen to reach the crack plane. Infiltrant was then forced via the tunnel into the crack. It was observed that epoxy resin tended to seep out of the widely opened crack before it became fully hardened. Experimental results agreed well with the computed results based on an $80 \%$ fill-up.

Figure 4(c) and (d) show the results from Ur-Rehman and Thomason ${ }^{5,14}$ using low-melting-point solder as infiltrant. The $R$ ratios were 0.05 and 0.3 , respectively. The specimen material was an EN 32B low-carbon steel. Ur-Rehman and Thomason ${ }^{5,14}$ mentioned an average proximity of the infiltrant to the crack tip of $0.5 \mathrm{~mm}$. They also reported a diamond pyramid hardness number of 24 for their low-melting-point solder. ${ }^{14}$ A yield strength of $78.5 \mathrm{MPa}$ was therefore employed for computation. In both cases, the computed values for the initial retardation were much lower than the experimentally observed value. Fractographs given in Ur-Rehman and Thomason ${ }^{5}$ and Ur-Rehman ${ }^{14}$ show that the infiltrated wedges were highly non-uniform through the specimen thickness. The point of the deepest penetration could be more than 2 to $3 \mathrm{~mm}$ closer to the crack tip than most of the other points along the front edge of the wedge. For an $R$ ratio of 0.05 [Fig. 4(c)], the discrepancy between computed and measured retardation responses may be attributed to this non-uniform penetration of the infiltrant. At the higher $R$ ratio of 0.3 [Fig. 4(d)], a larger crack opening displacement should alleviate the phenomenon of non-uniform penetration. However, the discrepancy between computed and measured initial retardation responses is even larger than that seen in Fig. 4(c). One possible explanation is the partial fill-up of crack opening as mentioned before. In fact, the computed retardation results assumed a $90 \%$ fill-up lie above the measured data [see Fig. 4(d)]. This suggests the actual fill-up ratio was somewhere between 90 and $100 \%$. In cases when molten metal is used as the infiltrant, considerable contraction due to solidification and the subsequent cooling down may also decrease the wedge thickness and contribute to a smaller observed retardation.

Figure 4(e) and (f) are also results by Ur-Rehman and Thomason ${ }^{5,14}$ using a low-viscosity ethylcyanoacrylate adhesive as infiltrant. The testing conditions were the same as that for Fig. 4(c) and (d). As the modulus and yield strength have not been reported, the data measured for epoxy resin were used as an approximation. For an $R$ ratio of 0.05 , computed results agree well with experimental data [Fig. 4(e)]. Results computed assuming fill-up percentages of 90 and $100 \%$ are shown in Fig. 4(f) for an $R$ ratio of 0.3 . The $90 \%$ fill-up case agrees well with experimental results. This is in line with previous observations and discussion that at a higher $R$ ratio, partial fill-up may result.

Figure 5(a) and (b) show the retarded growth responses in AISI 4130 alloy steel and 5083-O aluminium alloy on infiltration with epoxy reinforced with $1 \mu \mathrm{m}$ silicon carbide and alumina particles, respectively. ${ }^{11,18}$ In this work, the crack growth rate versus $\Delta K_{\text {eff }}$ relationship that was reported could not cover the whole range of $\Delta K_{\text {eff }}$ involved. Fortunately, the authors have also reported the fraction of crack opening $\left(\Delta K_{\text {eff }} / \Delta K\right)$. This allows the experimental $K_{\mathrm{op}} / K_{\max }$ data to be deduced and the latter are used for comparison with predictions in Fig. 5(a) and (b). Measured retardation is less than the computed values for AISI 4130. This may be attributed to highly non-uniform penetration of infiltrant through the thickness. In fact the fractograph reported in Song et al. ${ }^{11}$ showed that over most part of the infiltrated crack length, the wedge coverage only amounted to about half the specimen thickness. For the 5083-O specimen [Fig. 5(b)], computed results agree well with those measured. Incidentally, the fractograph for this case ${ }^{18}$ showed thorough penetration of the infiltrant.

Figure 5(c) and (d) shows the results by Sharp et al. ${ }^{6}$ using two low-viscosity epoxy resins on 7050-T73651 specimens. They used relatively low ISL values $[0.5$ for Fig. 5(c) and 0.8 for Fig. 5(d)]. Their work also differed with all previous works in that they employed a constant load range of $3.6 \mathrm{kN}$ instead of a constant $\Delta K$. No crack growth rate data nor growth rate versus $\Delta K_{\text {eff }}$ were provided in Sharp et al. ${ }^{6}$ Fortunately, a few raw compliance curves (load against crack mouth opening) have been included. These allow a limited amount of experimental $K_{\mathrm{op}} / K_{\max }$ data to be extracted approximately and compared with computed results. Yield strengths for the 

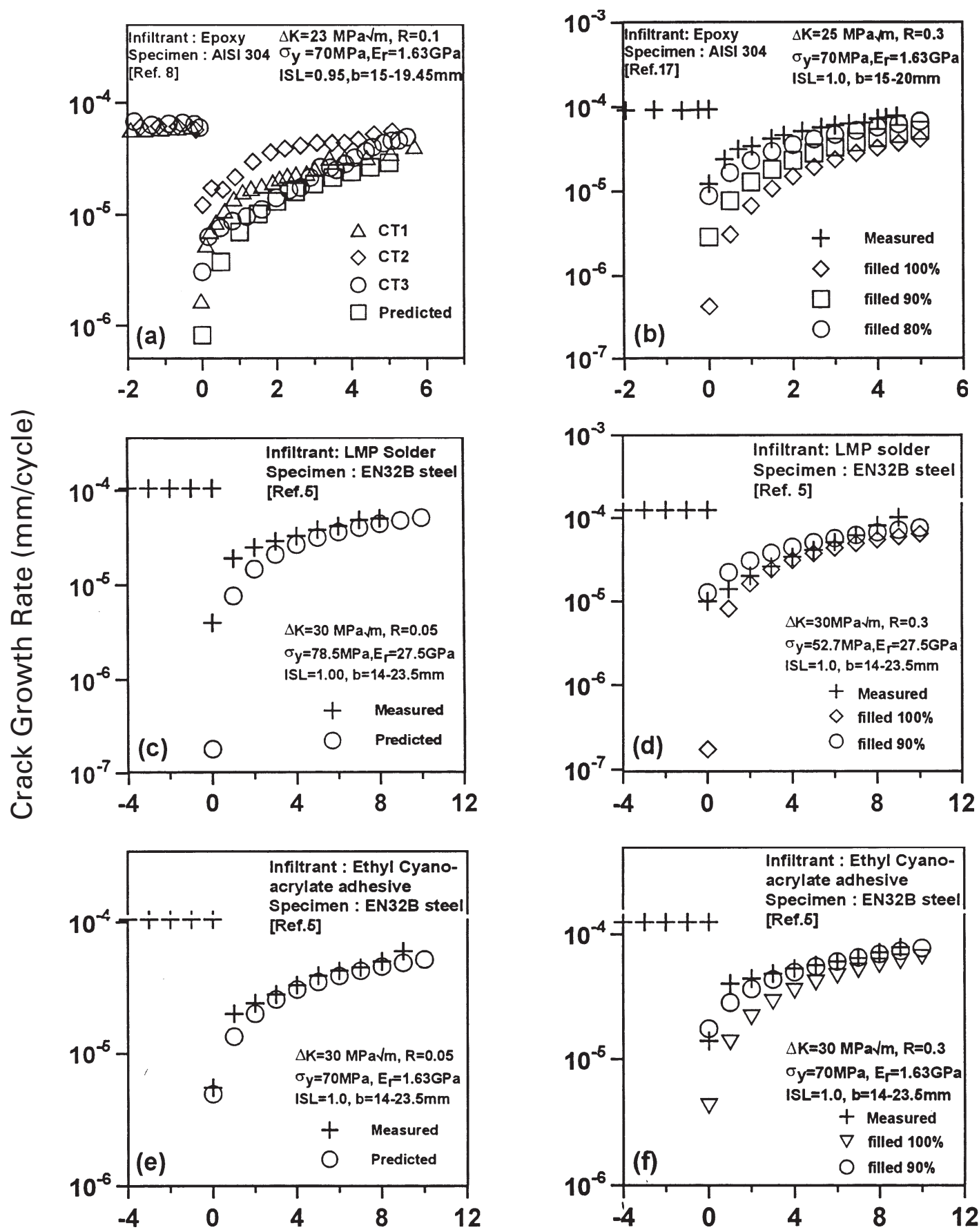

Distance From Infiltrated Crack Tip (mm)

Fig. 4 Comparison of predicted and observed retardation responses due to the infiltration of (a) epoxy in 304 stainless steel $(R=0.1)$; (b) epoxy in 304 stainless steel $(R=0.3)$; (c) low-melting-point solder in EN32B steel $(R=0.05)$; (d) low-melting-point solder in EN32B steel $(R=0.3)$; (e) ethylcyanoacrylate in EN32B steel $(R=0.05)$; (f) ethylcyanoacrylate in EN32B steel $(R=0.3)$.

epoxies are not available but the tensile strengths and Young's moduli have been reported. Polymers are somewhat stronger in compression than in tension ${ }^{19}$ and the yield strength in compression may be somewhat higher than the tensile strength. In the current computation, the yield strengths have been taken as the respective tensile strengths as well as some percentages above the tensile strengths. Figure 5(c) shows the case for ISL $=$ 0.5 with the epoxy having tensile strengths of 75 to $88 \mathrm{MPa}$. Computed results using yield strengths of 


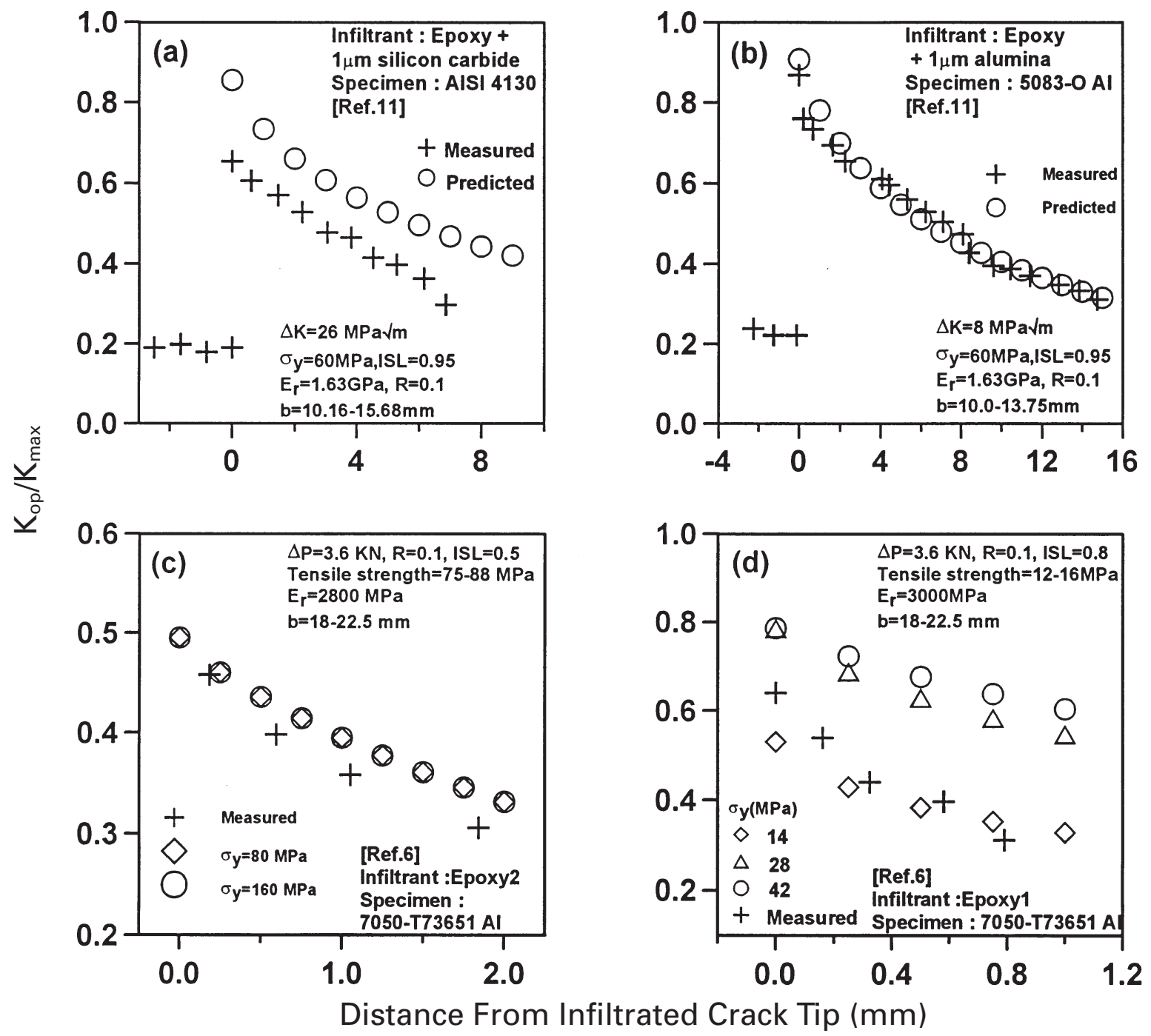

Fig. 5 Comparison of predicted and observed retardation responses due to the infiltration of (a) epoxy $+1 \mu \mathrm{m}$ silicon carbide in AISI 4130 steel $(\mathrm{R}=0.1)$; (b) epoxy $+1 \mu \mathrm{m}$ alumina in $5083-\mathrm{O} \mathrm{Al}(R=0.1)$; (c) a high-strength epoxy in 7050-T73651 $\mathrm{Al}$ (ISL $=0.5)$; (d) a lowstrength epoxy in 7050-T73651 $\mathrm{Al}(\mathrm{ISL}=0.8)$.

$80 \mathrm{MPa}$ and $160 \mathrm{MPa}$ show no significant difference. The observed $K_{\mathrm{op}} / K_{\max }$ data agree well with the computed results. Figure 5(d) shows the case for ISL $=0.8$ with the epoxy having tensile strengths of 12 to $16 \mathrm{MPa}$. Computed results start to show no significant difference when the yield strength is higher than $28 \mathrm{MPa}$. The observed $K_{\text {op }} / K_{\text {max }}$ data are close to the computed results based on a yield strength of $14 \mathrm{MPa}$.

\section{CONCLUSION}

A model for evaluating the development of crack closure following the infiltration of foreign material into a fatigue crack has been established. The induced crack closure will increase with the infiltrant being closer to the crack tip, with a higher infiltration stress level and with a higher fill-up ratio. A higher infiltrant yield strength and Young's modulus will also increase the amount of induced crack closure. However, such increase becomes insignificant when the infiltrant strength or modulus is above certain values. With an increase in cyclic loading ratio, the amount of induced crack closure first decreases and then increases. Growth behaviour prediction from the current model agrees well with a number of experimental observations collected from the literature.

\section{Acknowledgements}

The authors gratefully acknowledge the financial support provided by the National Science Council under the projects NSC-87-TPC-E-002-006 and NSC-88-2212E-002-057. 


\section{REFERENCES}

1 H. Kitagawa, S. Toyohira and K. Ikeda (1979) A new method of arresting fatigue crack growth by artificial wedge. In: Proceedings of International Conference on Fracture Mechanics in Engineering Applications, Bangalore, India (Edited by G. C. Sih and S. R. Valluri), Sijthoff and Noordhoff, The Netherlands, pp. 281-293.

2 R. S. Vecchio, J. S. Crompton and R. W. Hertzberg (1986) Anomalous aspects of crack closure. Int. 7. Fract. 31, R29-R33.

3 R. W. Hertzberg, C. H. Newton and R. Jaccard (1988) Crack closure: correlation and confusion. In: ASTM STP 982 (Edited by J. C. Newman, Jr and W. Elber), American Society for Testing and Materials, Philadelphia, PA, pp. 139-148.

4 C. S. Shin and S. H. Hsu (1992) Fatigue life extension by an artificially induced retardation mechanism. Engng Fract. Mech. $43,677-684$.

5 A. Ur-Rehman and P. F. Thomason (1993) The effect of artificial fatigue crack closure on fatigue crack growth. Fatigue Fract. Engng Mater. Struct. 16, 1081-1090.

6 P. K. Sharp, J. Q. Clayton and G. Clark (1997) Retardation and repair of fatigue cracks by adhesive infiltration. Fatigue Fract. Engng Mater. Struct. 20, 605-614.

7 C. S. Shin, C. M. Wang and P. S. Song (1996) Fatigue damage repair-a comparison of some possible methods. Int 7. Fatigue 18, 535-546.

8 C. S. Shin, K. C. Huang and R. Z. Li (1998) Artificial retardation of fatigue crack growth by the infiltration of cracks by foreign materials. Fatigue Fract. Engng Mater. Struct. 21, 835-846.

9 P. S. Song, C. M. Wang and C. S. Shin (1994) An investigation on various infiltration methods for fatigue crack repair. In: Proceedings of the 1994 Far East Conference on NDT (FENDT '94) and ROCSNT Ninth Annual Conference, Taipei, R.O.C., November 1994, (Edited by W. H. Chen, T. T. Wu and K. C. Wu), The Republic of China's Society for Nondestructive Testing, Taiwan, pp. 221-228.

10 B. C. Sheu, P. S. Song and C. S. Shin (1994) The effect of infiltration induced crack closure on crack growth retardation. Scripta Metallurgica Materialia 31, 1301-1306.

11 P. S. Song, S. Hwang and C. S. Shin (1998) Effect of artificial closure materials on crack growth retardation. Engng Fract. Mech. 60, 47-58.

12 C. S. Shin and Z. Z. Chen (1999) A novel method for repairing fatigue crack. Special issue on 'Recent Developments in Fatigue and Fracture Mechanics-based Analysis and Design of Structures', 7. Struct. Engng (Structural Engineering Research Centre, India) 26, 55-62.

13 Y. Y. Wang, M. X. Zho and D. Q. Feng (1993) A study of retarding fatigue crack growth using an artificial wedge. Fatigue Fract. Engng Mater. Struct. 16, 363-376.

14 A. Ur-Rehman (1992) An investigation of methods of reducing fatigue crack growth by artificial crack-closure effects. $\mathrm{PhD}$ Thesis, Department of Aeronautical and Mechanical Engineering, University of Salford.

15 S. Mall and J. C. Newman Jr (1985) The Dugdale model for compact specimen. In: ASTM STP 868 (Edited by M. F. Kanninen and A. T. Hopper), American Society for Testing and Materials, Philadelphia, PA, pp. 113-128.

16 B. Budiansky and J. W. Hutchinson (1978) Analysis of closure in fatigue crack growth. F. Appl. Mech. 45, 267-76.
17 R. Z. Li (1998) Investigation on artificially induced fatigue crack growth retardation. $\mathrm{PhD}$ Thesis, Department of Mechanical Engineering, National Taiwan University.

18 Y. P. Zhao (1995) Fatigue crack retardation by artificially induced crack closure. MS Thesis, Department of System Engineering, Chung Cheng Institute of Technology, ROC.

19 M. F. Ashby (1999) Materials Selection in Mechanical Design, 2nd edn. Butterworth Heinemann, Oxford.

\section{APPENDIX}

\section{STRESS INTENSITY AND COD CALCULATION UNDER PIN LOADING AND UNIFORM PRESSURE FOR CT SPECIMEN ${ }^{15}$}

\section{Pin-loaded holes with loading $\mathbf{P}$}

Stress intensity:

$K_{P}=\frac{p}{\sqrt{W}} F\left(\frac{a}{W}\right)$

where

$F(\alpha)$

$=\frac{(2+\alpha)\left(0.886+4.64 \alpha-13.32 \alpha^{2}+14.72 \alpha^{3}-5.6 \alpha^{4}\right)}{(1-\alpha)^{3 / 2}}$

$\alpha=\frac{a}{W}$

Crack opening displacement at a distance $c$ from the hole centreline:

$V_{P}=\frac{2 P}{E} \int_{c / W}^{\alpha} \frac{F(\alpha) G(\alpha, \Delta)}{\sqrt{2 \pi(\alpha-\mathrm{c} / W)}} \mathrm{d} \alpha$

where

$G(\alpha, \Delta)=\frac{\left(1+A_{1} \Delta+A_{2} \Delta^{2}\right)}{(1-\Delta)^{3 / 2}}$

$A_{1}=3.57+12.5(1-\alpha)^{8}$

$A_{2}=5.1-15.32 \alpha+16.58 \alpha^{2}-5.97 \alpha^{3}$

$\Delta=\frac{b}{W^{\prime}}=\frac{b}{W-a+b}$

The crack opening displacement at a distance $\xi$ measured from crack tip can also be calculated by the following equation:

$\frac{E V_{P}}{P}=4 \sqrt{\frac{\xi}{2 \pi W}}\left[1+C_{1}\left(\frac{\xi}{a}\right)+C_{2}\left(\frac{\xi}{a}\right)^{2}\right] F(\alpha)$

where

$C_{1}=-1.25+9.76 \alpha-20.15 \alpha^{2}+16.62 \alpha^{3}$ 
and

$C_{2}=0.64-4.34 \alpha+10.24 \alpha^{2}-8.46 \alpha^{3}$

Uniform pressure $\sigma$ on part of the crack surface (from $b_{1}$ to $b_{2}$ )

Stress intensity:

$K_{\sigma}=\int_{b_{2}}^{b_{1}} \frac{2 \sigma}{\sqrt{2 \pi b}} G(\alpha, \Delta) \mathrm{d} b$

or

$K_{\sigma}=\sigma \sqrt{\frac{W}{8 \pi}} H\left(\alpha, B_{1}, B_{2}\right)$ where

$H=\frac{1}{(1-\alpha)^{3 / 2}}$

$\times\left[\begin{array}{l}2 B\left(1+A_{1}+A_{2}\right) \sqrt{B^{2}+(1-\alpha) B} \\ +(1-\alpha)\left(5+A_{1}-3 A_{2}\right) \sqrt{B^{2}+(1-\alpha) B} \\ +(1-\alpha)^{2}\left(3-A_{1}+3 A_{2}\right) \ln (\sqrt{B}+\sqrt{B+1-\alpha})\end{array}\right] \mid \begin{aligned} & B=B_{1} \\ & B=B_{2}\end{aligned}$

$B_{j}=\frac{b_{j}}{W}$

Crack opening displacement at a distance $c$ from hole centreline:

$V_{\sigma}=\frac{\sigma W}{2 \pi E} \int_{c / W}^{\alpha} \frac{H\left(\alpha, B_{1}, B_{2}\right) G(\alpha, \Delta)}{\sqrt{\alpha-c / W}} \mathrm{~d} \alpha$ 